\title{
Characterization and Functional Analysis of Pyrabactin Resistance-Like Abscisic Acid Receptor Family in Rice
}

\author{
Xiaojie Tian ${ }^{1,2 \dagger}$, Zhenyu Wang ${ }^{1 \dagger}$, Xiufeng Li ${ }^{1,2}$, Tianxiao Lv ${ }^{1,2}$, Huazhao Liu ${ }^{1,3}$, Lizhi Wang $^{4}$, Hongbin Niu ${ }^{* *}$ \\ and Qingyun Bu*
}

\begin{abstract}
Background: Abscisic acid (ABA) plays crucial roles in regulating plant growth and development, especially in responding to abiotic stress. The pyrabactin resistance-like (PYL) abscisic acid receptor family has been identified and widely characterized in Arabidopsis. However, PYL families in rice were largely unknown. In the present study, 10 out of 13 PYL orthologs in rice (OsPYL) were isolated and investigated.

Results: Quantitative reverse transcription-polymerase chain reaction (qRT-PCR) analysis showed that expression of OsPYL genes is tissue-specific and display differential response to ABA treatment, implying their functional diversity. The interaction between 10 OsPYL members and 5 protein phosphatase $2 \mathrm{C}$ in rice (OsPP2C) members was investigated in yeast two-hybrid and tobacco transient expression assays, and an overall interaction map was generated, which was suggestive of the diversity and complexity of ABA-sensing signaling in rice. To study the biological function of OsPYLS, two OsPYL genes (OsPYL3 and OsPYL9) were overexpressed in rice. Phenotypic analysis of OsPYL3 and OsPYL9 transgenic rice showed that OsPYLs positively regulated the ABA response during the seed germination. More importantly, the overexpression of OSPYL3 and OsPYL9 substantially improved drought and cold stress tolerance in rice.
\end{abstract}

Conclusion: Taken together, we comprehensively uncovered the properties of OsPYLs, which may be good candidates for the improvement of abiotic stress tolerance in rice.

Keywords: Rice, ABA receptors; PYL; Drought stress; Cold stress; Seed germination

\section{Background}

Abscisic acid (ABA) plays pivotal roles in regulating plant growth and development, including seed dormancy, germination, and seedling growth. More importantly, $\mathrm{ABA}$ is the key phytohormone that functions in a plant's response to abiotic stressors such as drought, high salinity, and extreme temperature (Cutler et al. 2010). During abiotic stress, ABA biosynthesis is activated, resulting in an increase in ABA levels in the plant. $\mathrm{ABA}$ binds to the pyrabactin resistant-like/regulatory

\footnotetext{
*Correspondence: newhobby@163.com; buqingyun@iga.ac.cn

${ }^{\dagger}$ Equal contributors

${ }^{5}$ National Engineering Research Centre for Wheat, Henan Agricultural University, Zhengzhou 450002, China

${ }^{1}$ Northeast Institute of Geography and Agroecology, Key Laboratory of Soybean Molecular Design Breeding, Chinese Academy of Sciences, Harbin 150081, China

Full list of author information is available at the end of the article
}

components of ABA receptors, PYL/RCAR (hereafter referred to as PYLs for simplicity), an ABA receptor family that promotes the interaction between PYL with protein phosphatase $2 \mathrm{C}$ ( $\mathrm{PP} 2 \mathrm{C})$, which then results in the release of SNF1-related protein kinase (SnRK)) from the repression of PP2C. Finally, the active SnRK phosphorylates and activates downstream transcriptional factors that promote the expression of ABA-regulated genes. An ABA signal is then generated, which in turn results in the acquisition of abiotic stress resistance in plants (Ma et al. 2009; Park et al. 2009; Cutler et al. 2010; Klingler et al. 2010). ABA receptor PYL proteins, which contain a conserved steroidogenic acute regulatory-related lipid transfer (START) protein domain, are the core components of this ABA sensing signaling pathway (Ma et al. 2009; Park et al. 2009). To date, PYL proteins have been identified from distinct plant species, including Arabidopsis,

\section{实 Springer}

(C) 2015 Tian et al. Open Access This article is distributed under the terms of the Creative Commons Attribution 4.0 International License (http://creativecommons.org/licenses/by/4.0/), which permits unrestricted use, distribution, and reproduction in any medium, provided you give appropriate credit to the original author(s) and the source, provide a link to the Creative Commons license, and indicate if changes were made. 
rice, tomato, and soybean, which present highly conserved PYL-mediated ABA-sensing signaling pathways (Ma et al. 2009; Park et al. 2009; Kim et al. 2012; Bai et al. 2013; Gonzalez-Guzman et al. 2014; He et al. 2014). The biochemical property and structure of PYLs have been extensively studied in the dicot plant model Arabidopsis (Ma et al. 2009; Melcher et al. 2009; Klingler et al. 2010; JoshiSaha et al. 2011). Some AtPYLs are monomers that facilitate interactions with $\mathrm{PP} 2 \mathrm{C}$ in the absence of $\mathrm{ABA}$; some AtPYLs are in a dimeric state and require $\mathrm{ABA}$ to form a complex with PP2C (Hao et al. 2011). Recent studies have investigated the biological functions of various AtPYLs. The overexpression of AtPYL5 leads to ABA hypersensitivity during early seedling development, as well as enhanced drought stress tolerance (Klingler et al. 2010). AtPYL8 is involved in root growth and development, which is in line with its root-specific expression pattern (Antoni et al. 2013). Although AtPYL13 is not an ABA receptor, it can positively regulate the $A B A$ signaling pathway by interacting with and inhibiting both the PYL receptors and the PP2C co-receptors (Li et al. 2013; Zhao et al. 2013). AtPY$\mathrm{L} 4{ }^{\mathrm{A} 194 \mathrm{~T}}$ forms stable complexes with PP2CA in the absence of ABA, and the overexpression of AtPYL4 $4^{\mathrm{A} 194 \mathrm{~T}}$ increases a plant's sensitivity to ABA-mediated inhibition of germination and seedling establishment, as well as enhances drought resistance (Pizzio et al. 2013). In addition, AtPYLs are largely functionally conserved, and the analysis of higher order mutants have indicated that AtPYLs regulate stomatal conductance (Gonzalez-Guzman et al. 2012).

Unlike AtPYLs, information on PYL homologs in rice is limited. Studies have predicted that rice has 13 OsPYL members that share high sequence similarity with AtPYLs (Kim et al. 2012; He et al. 2014). Core components in ABA signaling, including OsPYLs, OsPP2C, OsSAPK2, and OsOREB1, have been identified in rice, and the ABA signaling transduction pathway has also been reconstituted in a protoplast system (Kim et al. 2012). The biochemical properties and structure of OsPYLs have also been recently reported (He et al. 2014). However, to date, only OsPYL/RCAR 5 has been identified as a positive regulator in seed germination, early seedling growth, and drought and salt stress tolerance (Kim et al. 2012; Kim et al. 2014). Other features of OsPYLs such as expression pattern, subcellular localization, interaction specificity with OsPP2C, and biological function, have not been examined.

The present study examined the tissue-specific expression pattern and distinct response of OsPYL members to ABA treatment, which was suggestive of its differential biological function. An overall interaction map between 10 OsPYLs and 5 OsPP2C members indicated that OsPYLs were selective of their interaction partner, thus indicating the complexity and specificity of the rice ABA-sensing signaling pathway. Furthermore, using
OsPYL3 and OsPYL9, we determined that OsPYLs play pivotal roles in ABA-mediated inhibition of seed germination, as well as drought and cold stress tolerance, which thereby could serve as potential targets for the improvement of abiotic stress tolerance in rice.

\section{Results}

\section{Expression Pattern of OsPYL Members}

Using the AtPYLs sequence as queries and search in the rice genome database, a total of 13 OsPYL orthologs were identified in rice. Among these, three (OsPYL11-13) are thought to be nonfunctional that were caused by a large fragment deletion in the $\mathrm{N}$ or C terminal of the gene (Kim et al. 2012). To obtain an overview of the expression pattern of OsPYL members in different tissues, quantitative RT-PCR was performed. Because of the high level of sequence similarity among OsPYL members, it was difficult to design primers that could discriminate between OsPYL2 and OsPYL9, and OsPYL7 and OsPYL8. Most of the OsPYLs were detected in all tissues, although differentially expressed (Fig. 1a). OsPYL7/8 was upregulated in embryos, OsPYL3 and OSPYL5 were upregulated in leaves, OsPYL1 in roots, and OsPYL2/9 in all tissues (Fig. 1a). These diverse tissue-specific patterns of OSPYLs were indicative of its diverse biological functions.

OsPYLs are also differentially expressed after ABA treatment (Fig. 1b). Some OsPYLs were downregulated such as OsPYL1, OsPYL2/9, and OsPYL3 (Fig. 1b), whereas OsPYL4 was upregulated (Fig. 1b). The expression of OsPYL5, OsPYL7/8, and OsPYL10 was not affected by ABA treatment (Fig. 1b). These findings suggest that $O s P Y L$ members play diverse roles in sensing the ABA signal.

\section{OsPYLs are Localized in the Cytosol and Nucleus}

To determine the subcellular localization of OsPYLs, green fluorescent protein (GFP)-OsPYL fusion proteins driven by a $35 \mathrm{~S}$ promoter were transiently expressed in Nicotiana benthamiana leaves. All tested OsPYLs were localized in both the cytosol and nucleus (Fig. 2a), which were consistent with the results involving AtPYL9 and soybean GmPYL members (Ma et al. 2009; Bai et al. 2013). These results indicated that PYLs of different plant species are localized in the same cellular regions, which might partly explain the functional conservation of PYL proteins as ABA receptors.

\section{OsPYL Members Selectively Interact with OsPP2C Members}

PYL family proteins, as functional ABA receptors, interact with clade A PP2Cs to form PYL-ABA-PP2C triple complexes that facilitate in the transmission of $A B A$ signals 


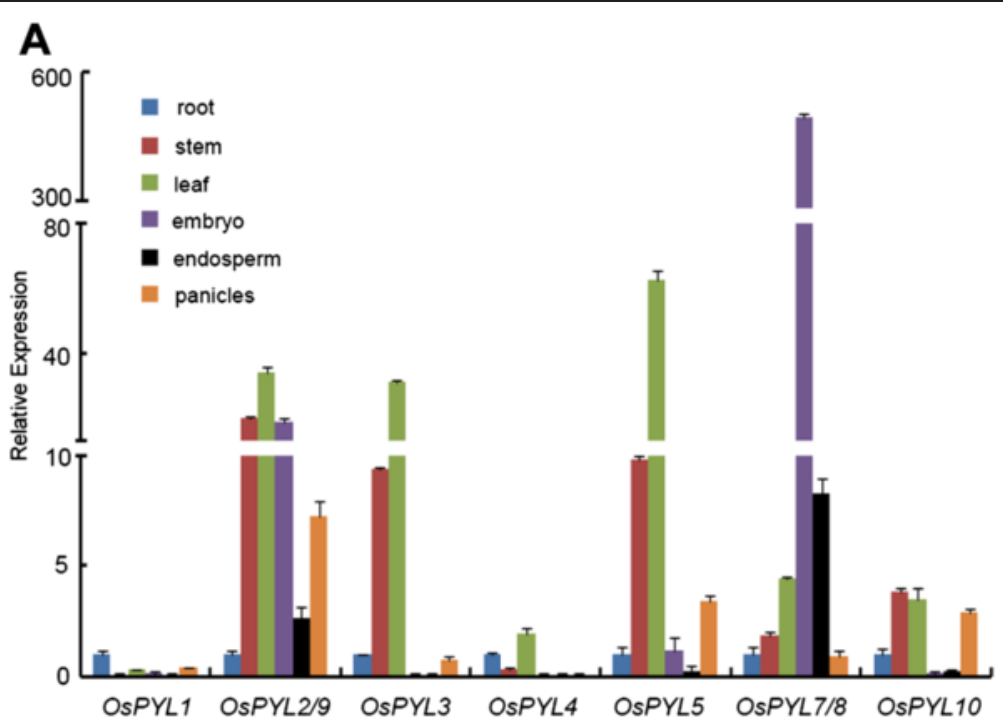

B

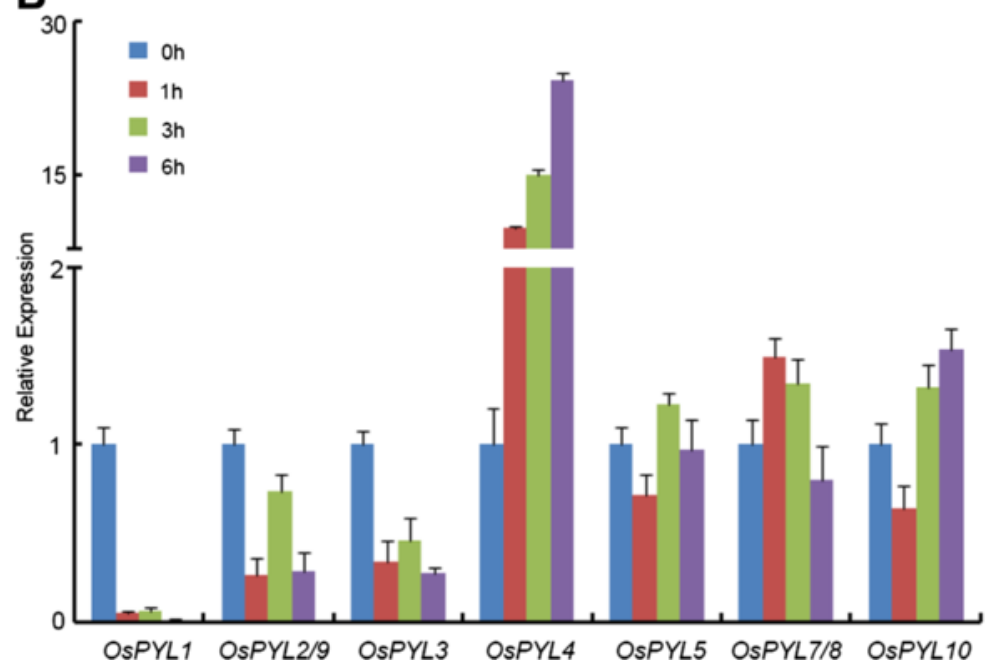

Fig. 1 Tissue-specific and ABA-regulated expression of OsPYLs genes. a Tissue-specific expression of OsPYLs was analyzed by quantitative RT-PCR in roots, leaves, stems, embryo, endosperm and panicles. The expression level of OsPYLs in roots was set as 1 and the fold change was analyzed via the $2^{-\Delta \Delta C T}$ method using the rice ubiquitin gene as an internal control. Values represent the mean \pm SD of three biological replicates. b ABA-regulated expression of OsPYLs was analyzed by quantitative RT-PCR. Two-week-old seedlings were incubated in liquid MS medium containing $200 \mu \mathrm{M}$ ABA for $0,1,3$ and $6 \mathrm{~h}$. The expression level of OsPYLs at $0 \mathrm{~h}$ was set as 1 and the fold change was analyzed via the $2^{-\triangle \triangle C T}$ method using the rice ubiquitin gene as an internal control. Values represent the mean \pm SD of three biological replicates

(Cutler et al. 2010; Joshi-Saha et al. 2011). A total of 10 predicted clade A OsPP2Cs have been identified in rice (Xue et al. 2008). In the present study, five clade A OsPP2Cs were isolated and investigated (Fig. 2b). To determine whether OsPYLs interacted with these OsPP2Cs, yeast two-hybrid assays were conducted in the absence or presence of ABA. The results showed that the interaction between OsPYLs and OsPP2Cs was selective and specific (Fig. 3, Additional file 1: Figure S1). Some OsPYLs interacted with all OsPP2C members, except for OsPYL2 and OsPYL10. Most interactions were ABA-dependent or ABA-enhanced. A few interactions were ABA-independent or constitutive such as that observed with OsPYL1 and OsPP2C53 (Fig. 3). Most OsPYLs strongly interacted with OsPP2C30 and OsPP2C53 in an ABA-dependent or ABAindependent manner, respectively (Fig. 3). These results demonstrated that OsPYLs bind to OsPP2Cs in diverse fashions and with different intensities. To confirm these interactions in plant cells, BiFC experiments in N. benthamiana leaves were performed. The in vivo interaction results indicated that these could reproduce, as well as validated the results of the yeast two-hybrid assay (Fig. 4). Based on the results of the yeast two-hybrid and BiFC assays, an overall interaction map was generated between the 10 

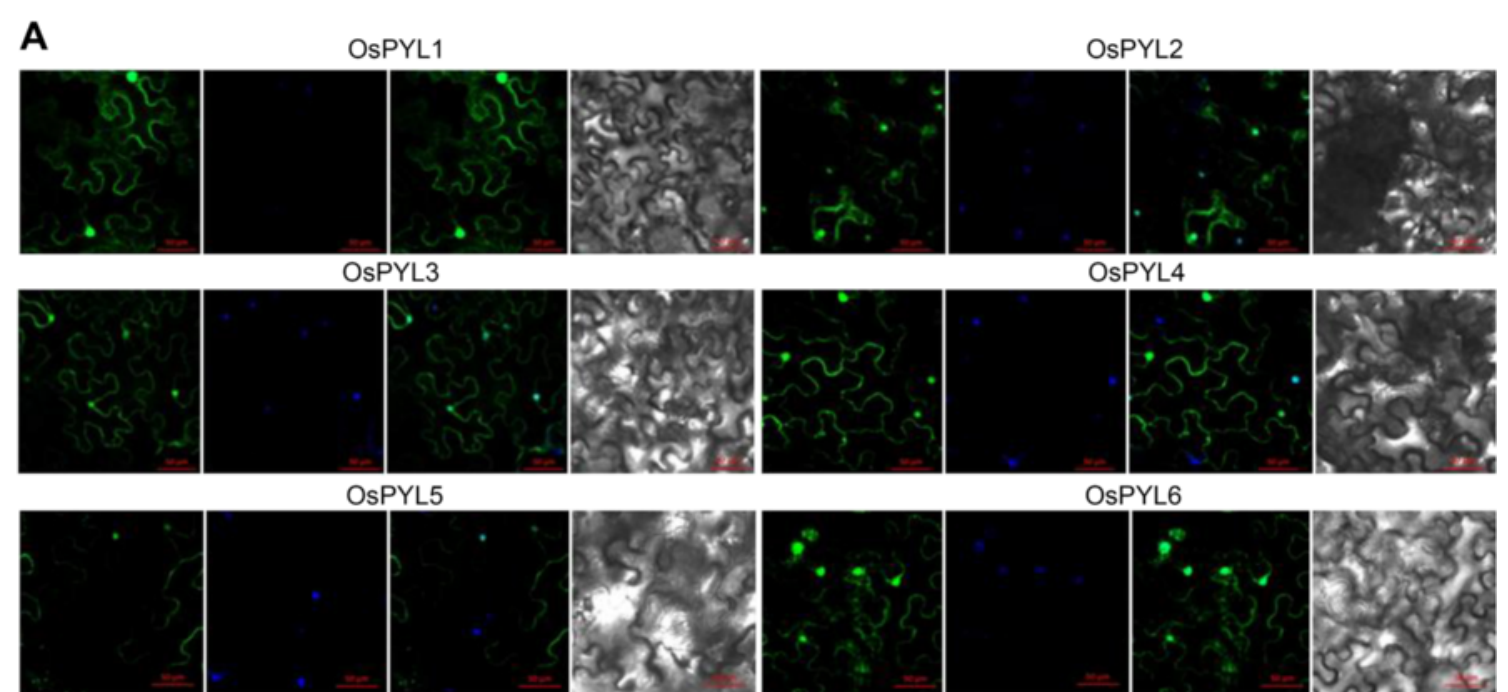

OsPYL5

OsPYL6
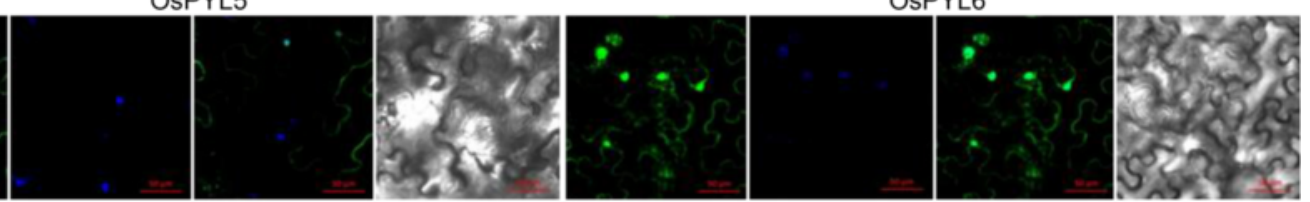

OsPYL7
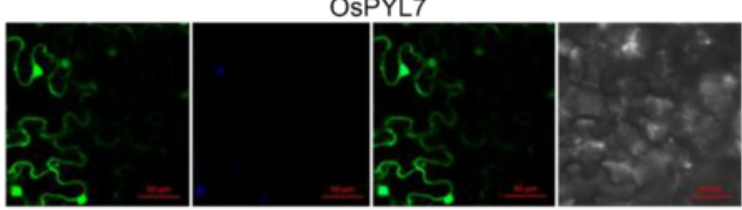

OsPYL8

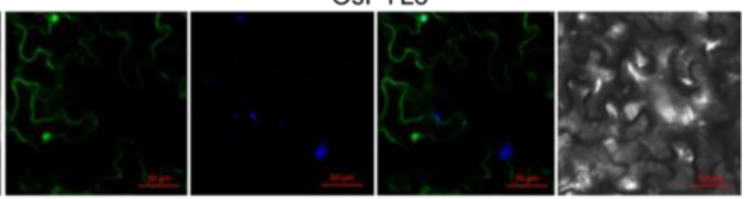

OSPYL9

OsPYL10
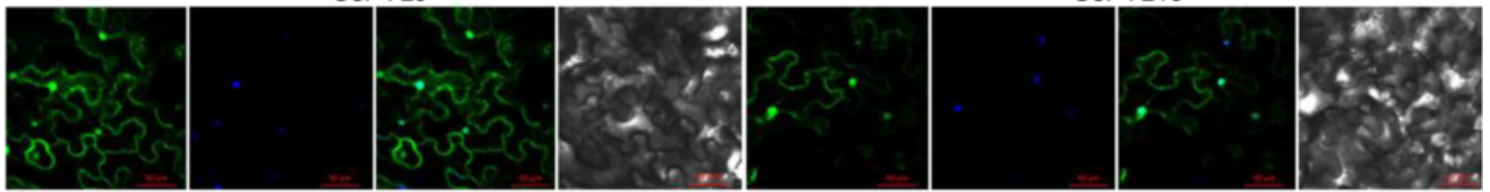

GFP

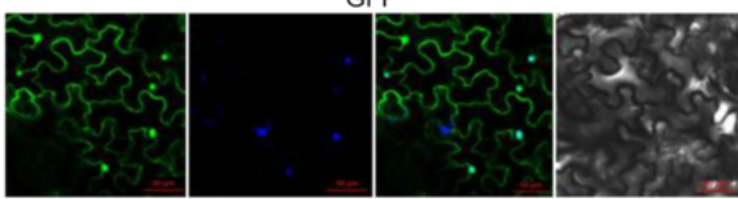

B

OsPP2C08

OsPP2C30
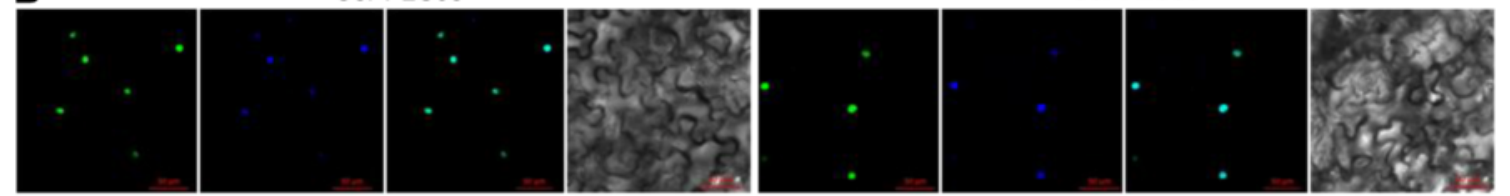

OsPP2C51

OsPP2C53
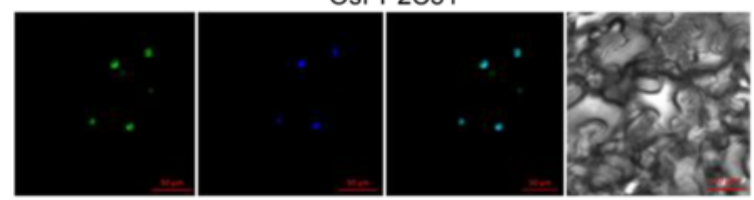

OsPP2C68
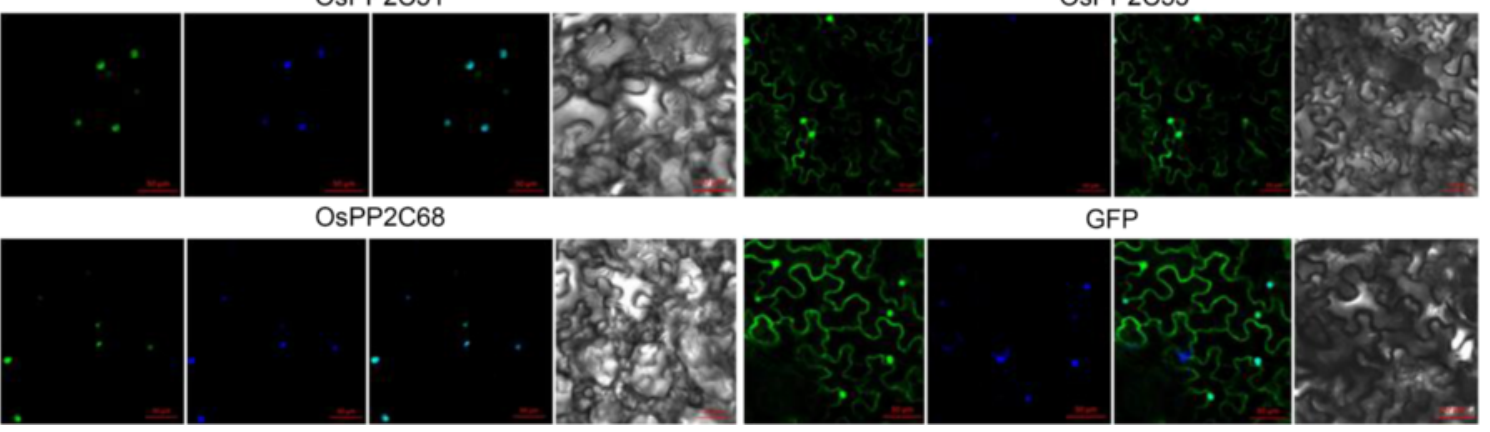

GFP
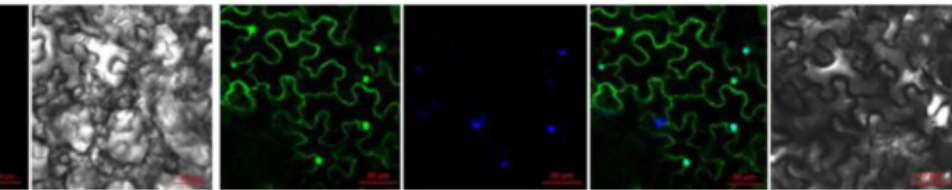

Fig. 2 (See legend on next page.) 
(See figure on previous page.)

Fig. 2 Subcellular localization of OsPYLs and OsPP2Cs. Confocal images were taken from Nicotiana benthamiana leaves epidermal cells. Constructs of (GFP)-OsPYLs (a) and (GFP)-OsPP2Cs (b) driven by the 355 promoter were infiltrated and observed at 3 days later. From left panel to right panel are GFP image, DAPI dye image, merged image and bright-field image. Empty GFP vector was used as control. The positions of nuclei were shown by DAPI staining

OsPYLs and 5 OsPP2C members (Fig. 3). These results provide evidence that the tested OsPYLs were capable of functioning as $\mathrm{ABA}$ receptors, and that the ABA-sensing mechanism was conserved among different plants.

\section{OsPP2C Determines the Subcellular Localization of the} OsPYLs-OsPP2C Complex in an ABA-independent Manner The results of the BiFC assay indicated that the subcellular localization of the fused green fluorescent proteins varied among different OsPYLs and OsPP2C members, and most of the OsPYL-OsPP2C complexes were localized in the nucleus (Fig. 4), which is not consistent with the localization of OsPYLs (Fig. 2a). To explore the underlying mechanism of this activity, the subcellular localization of five OsPP2C members was investigated. OsPP2C53 was localized to both the nucleus and cytosol, whereas other OsPP2C members were detected only in the nucleus (Fig. 2b). Comparative analysis showed a similarity between the subcellular localization of OsPP2C members and that of OsPYLs-OsPP2C complexes (Figs. $2 \mathrm{~b}$ and 4 ). In the $\mathrm{BiFC}$ assay, $\mathrm{ABA}$ was also injected to test whether

\begin{tabular}{cccccccccccc}
\hline & OsPP2C08 & OsPP2C30 & OsPP2C51 & OsPP2C53 & OsPP2C68 \\
\hline OsPYL1 & $\times$ & $* * *$ & $\times$ & $* * * *$ & $\times$ & $* * * *$ & $* * * *$ & $* * * *$ & $* * * *$ & $* * * *$ \\
OSPYL2 & $\times$ & $\times$ & $\times$ & $\times$ & $\times$ & $\times$ & $*$ & $*$ & $\times$ & $*$ \\
OSPYL3 & $* *$ & $* *$ & $* * * *$ & $* * * *$ & $*$ & $* *$ & $* * * *$ & $* * * *$ & $\times$ & $* * *$ \\
OSPYL4 & $\times$ & $*$ & $*$ & $* * * *$ & $\times$ & $\times$ & $* * * *$ & $* * * *$ & $\times$ & $* * * *$ \\
OsPYL5 & $*$ & $* *$ & $*$ & $* * * *$ & $*$ & $*$ & $* * * *$ & $* * * *$ & $\times$ & $* * * *$ \\
OsPYL6 & $*$ & $*$ & $*$ & $* * * *$ & $*$ & $*$ & $* * * *$ & $* * * *$ & $*$ & $* * *$ \\
OSPYL7 & $*$ & $*$ & $*$ & $*$ & $*$ & $*$ & $*$ & $* * * *$ & $\times$ & $\times$ \\
OSPYL8 & $*$ & $* * *$ & $*$ & $* * * *$ & $*$ & $* * *$ & $* * * *$ & $* * * *$ & $\times$ & $* *$ \\
OSPYL9 & $\times$ & $*$ & $*$ & $* * * *$ & $\times$ & $*$ & $\times$ & $* * * *$ & $\times$ & $*$ \\
OSPYL10 & $\times$ & $*$ & $\times$ & $\times$ & $\times$ & $\times$ & $*$ & $* * * *$ & $\times$ & $*$ \\
\hline
\end{tabular}

Fig. 3 Interaction map between OsPYLs and OsPP2Cs. Interaction between OsPYLs and OsPP2Cs was first examined in yeast two-hybrid, "-" and " + " indicated without and with ABA supplement. Number of "**" indicate the interaction strength from weak to strong, one $\left(^{*}\right)$ to four star $\left({ }^{* * *}\right)$ represent that the yeast colony can grow on SD/-His /-Leu/-Trp plate containing different concentration of 3-AT (0, 3.0, 5.0 and $10 \mathrm{mM}$ ). " $x$ " means no interaction. These interactions were further confirmed by BiFC in tobacco transient expression system
ABA affected the interaction between OsPYLs and OsPP2Cs. The results showed that ABA treatment only enhanced interaction strength, and did not affect the subcellular localization of the OsPYL-OsPP2C complexes (Fig. 4). Consequently, the findings indicated that OsPP2C members can determine the subcellular localization of OsPYLOsPP2C complexes in an ABA-independent manner.

\section{Overexpression of OsPYL3 and OsPYL9 Confers ABA Hypersensitivity during Seed Germination}

To determine whether OsPYLs are functional ABA receptors in rice, OsPYL3 and OsPYL9 were selected for further investigation. Constructs of OsPYL3 and OsPYL 9 driven by the $35 S$ promoter were transformed into rice, and more than 20 independent transgenic lines were produced for each gene. Three independent transgenic lines of each gene were chosen for further analysis. The expression levels of OsPYL3 and OsPYL9 in the transgenic lines were measured by quantitative RT-PCR and RT-PCR, respectively (Fig. $5 \mathrm{a}$ and b). OsPYL3 and OsPYL9 were overexpressed in the transgenic lines compared to the control lines (Fig. $5 \mathrm{a}$ and $\mathrm{b}$ ). A seed germination assay was conducted to examine the ABA-related phenotype of OsPYL3 and OsPYL9 overexpression lines. In the absence of ABA, OsPYL3 and OSPYL9 overexpression lines showed a slight delay in germination rate, and the OsPYL9 overexpression line germinated at a slower rate compared to that of the OsPYL3 overexpression line (Fig. 5c, d, and g). Application of different concentrations of ABA showed that the seed germination rate of the OsPYL3 and OsPYL9 overexpression lines was markedly slower than that of the controls (Fig. 5c, e, f, h, and i). OsPYL9 overexpression lines hardly germinated in a medium containing $3 \mu \mathrm{M}$ of ABA until 5 days later (Fig. $5 \mathrm{i}$ ). This finding indicated that the overexpression of OSPYL3 and OsPYL9 conferred ABA-hypersensitivity during seed germination and functioned as an active ABA receptor. In addition, a higher expression level of OSPYL9 than that observed with OsPYL3 in embryos may explain its enhanced phenotype during ABA-regulated seed germination (Figs. 1a and 5).

\section{Overexpression of OsPYL3 and OsPYL9 Enhances Drought Stress Tolerance}

To further investigate the biological function of OsPYLs, OsPYL3 and OsPYL9 overexpression lines were subjected to a drought tolerance assay. Three-week-old seedlings of 


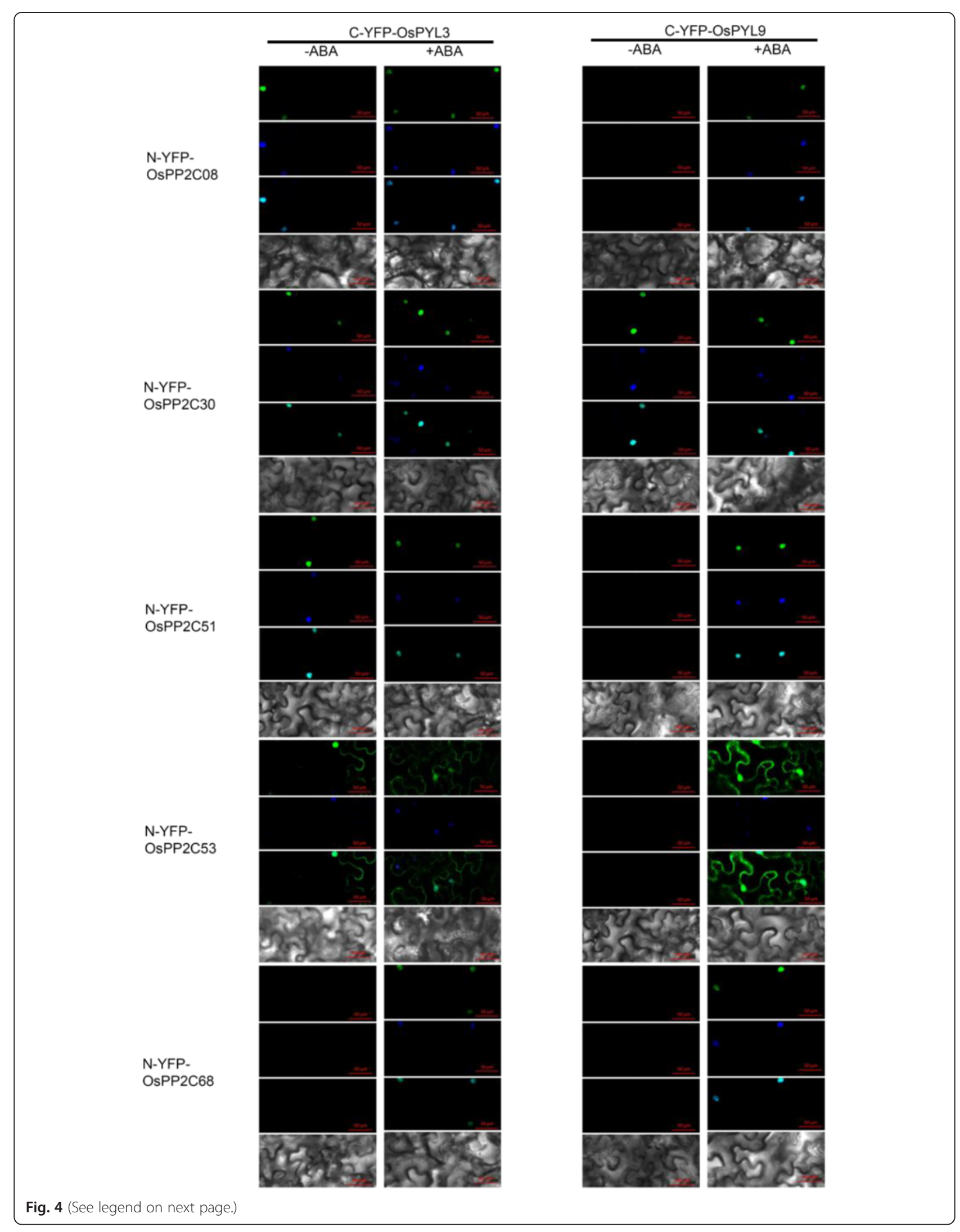


(See figure on previous page.)

Fig. 4 OsPYL3 and OsPYL9 interact with OsPP2Cs in planta. In planta interaction and subcellular localization analysis using agroninfiltrated Nicotiana benthamiana leaves. The interaction was detected by fluorescence in BiFC analysis. $100 \mu \mathrm{M}$ ABA was injected at $24 \mathrm{~h}$ before observation. From upper panel to bottom panel are YFP image, DAPI dye image, merged image and bright-field image. The positions of nuclei were shown by DAPI staining

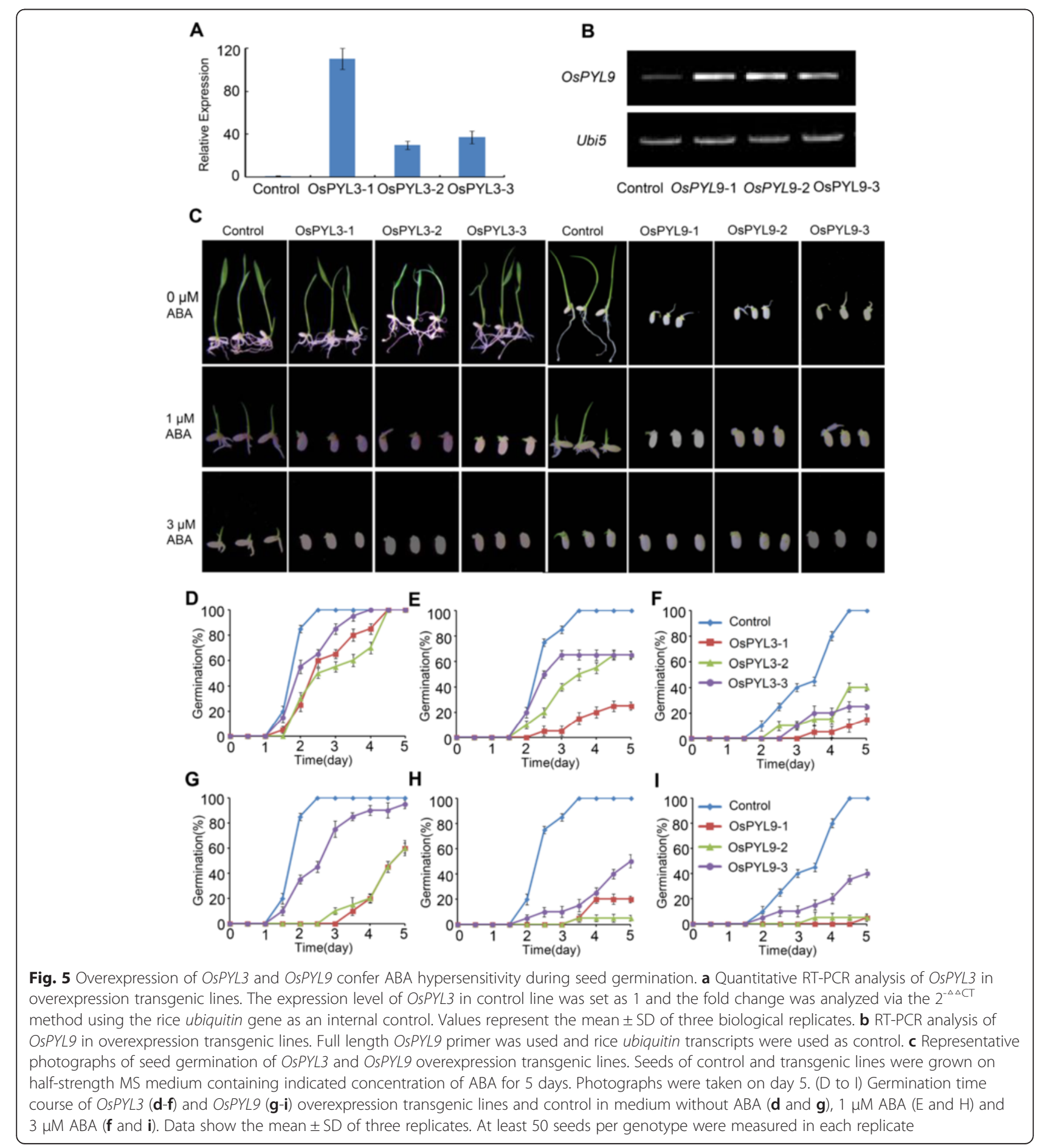


similar sizes showed the capability of withholding water for 10 days, control seedling leaves were curved and wilted and its seedling had fallen down, whereas OsPYL3 and OsPYL9 overexpression lines remained upright (Fig. 6a). After 7 days of re-watering, over $80 \%$ of the OsPYL3 and
OsPYL9 overexpression lines were turgid and survived; in contrast, only less than $10 \%$ of the control lines remained alive (Fig. $6 \mathrm{a}-\mathrm{c}$ ). These results indicated that the ectopic expression of OsPYL3 and OsPYL9 enhanced drought stress tolerance in rice (Fig. 6a-c).

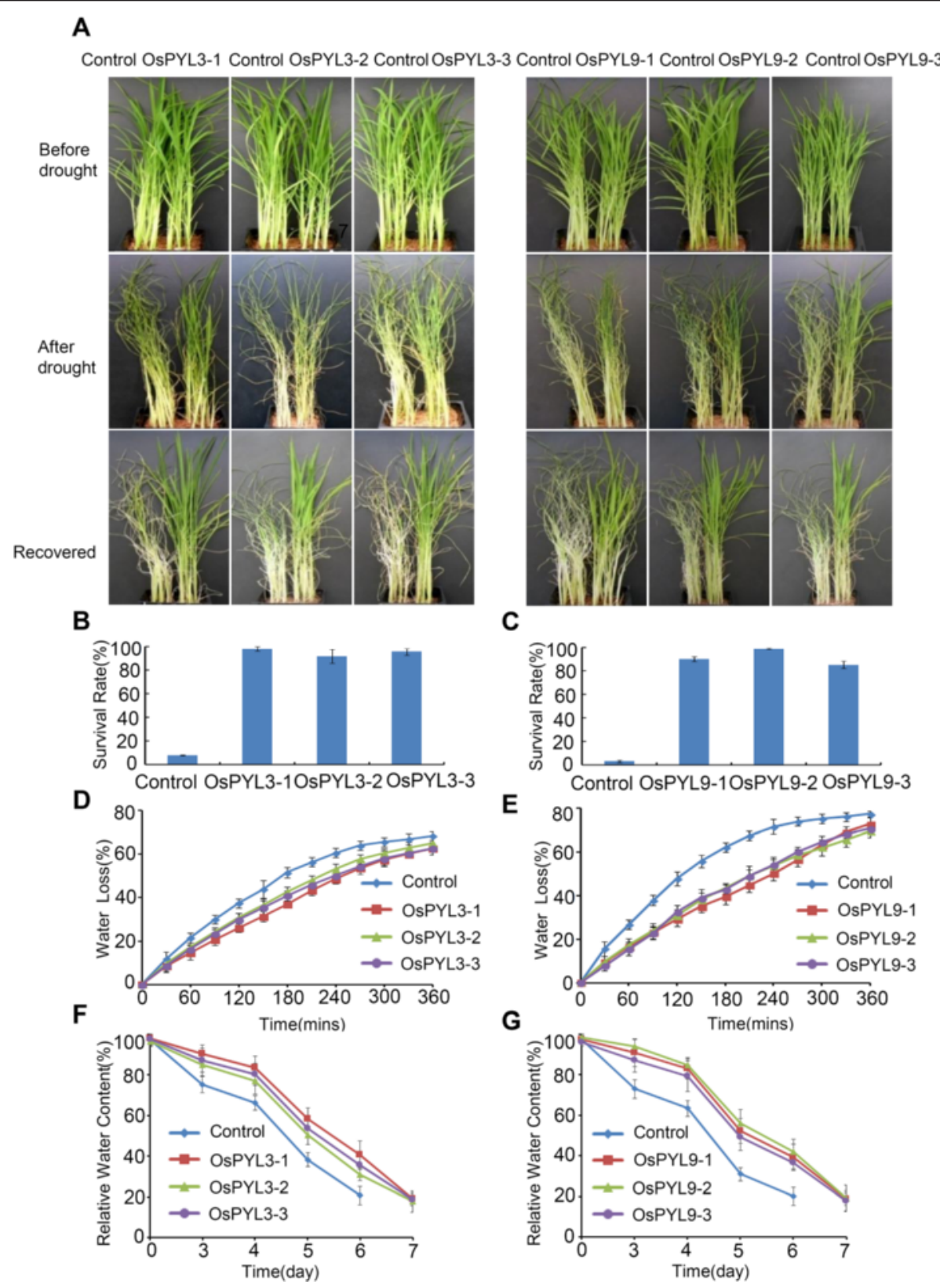

Fig. 6 Overexpression of OSPYL3 and OSPYL9 exhibit enhanced drought stress tolerance. a Three-week-old OsPYL3 and OsPYL9 overexpression seedlings were subjected to drought conditions by withholding water and then rewatered. Photographs were taken before drought (upper panel) and 10 dayays after drought (middle panel), and 7 days after rewatering (bottom panel). b and c Survival rates of the drought treated OsPYL3 (B) and OsPYL9 (C) overexpression seedlings after 7 days of rewatering. Values are mean \pm SD ( $n=30$ for each replicate) of three independent experiments. $\mathbf{d}$ and $\mathbf{e}$ Water loss rate of OSPYL3 $\mathbf{d}$ and OSPYL9 (e) overexpression lines. Leaves of the same developmental stages were excised and weighed at various time points after detachment. Values are means \pm SD of three individual plants per genotype. Experiments were repeated at least three times with similar results. ( $\mathbf{f}$ and $\mathbf{g}$ ) Relative water content of OSPYL3 ( $\mathbf{f})$ and OSPYL9 $(\mathbf{g})$ overexpression lines. Seedling of similar stage was withhold water for indicated days and used for measure relative water content. Values are means \pm SD of three individual plants per genotype. Experiments were repeated three times 
Plants lose water mainly through stomata transpiration. To determine whether stomatal closure is involved in the enhanced drought tolerance of OsPYL3 and OsPYL9 overexpression lines, a water-loss assay was performed using detached leaves. The water loss rate of the OsPYL3 and OsPYL9 overexpression lines was significantly slower than that observed in the controls (Fig. 6d and e). Relative water content (RWC) more accurately reflects the physiological consequence of cellular water deficit. Fig. 6e and g shows that with drought stress, the OsPYL overexpression lines lost water at a slower rate and have a lower minimum relative water content that helped the plants survive during severe drought conditions. These results indicated that the OSPYL3 and OsPYL9 overexpression lines have a faster rate of stomatal closure and were hypersensitive to drought treatment compared to the controls, thereby contributing to a high level of drought stress tolerance.

\section{Overexpression of OsPYL3 and OsPYL9 Increases Cold Stress Tolerance}

The rice seedlings were also subjected to cold stress. To examine whether OsPYLs played a role during cold stress response, a cold tolerance assay was performed. Two-week-old rice seedlings of similar size were incubated in a $10{ }^{\circ} \mathrm{C}$ growth chamber for 4 days. After 4 days, the leaves of the transgenic lines remained green and flats, and only the leaf tips were rolled up. In contrast, the leaves of the control lines were wilted, rolled up, and dry (Fig. 7a). After 7 days of recovery at the normal temperature, the survival rate of the OsPYL3 and OsPYL 9 transgenic plant was $>50 \%$, compared to the $>95 \%$ death rate of the control lines (Fig. 7a-c). To evaluate the effect of cold stress on cell membranes, two-week-old rice seedlings were exposed to a $10{ }^{\circ} \mathrm{C}$ environment, and relative ion leakage was measured. Without cold treatment, the relative ion leakage rate of the transgenic lines and control lines was similar. After 3 days cold treatment, the relative ion leakage rate of the control lines was $70 \%$, whereas that of the OsPYL3 and OsPYL9 overexpression lines was $<10 \%$ (Fig. $7 \mathrm{~d}$ and e). These findings indicated that the increased cell membrane stability of OsPYL3 and OsPYL9 overexpression lines during cold stress partially, if not totally, contributed to the observed enhanced tolerance of cold stress. These results indicated that overexpression of OsPYL3 and OSPYL9 substantially improved cold stress tolerance in rice.

\section{Expression of ABA-Regulated Genes is Enhanced in OsPYL3 and OsPYL9 Overexpression lines}

Because OSPYL3 and OSPYL9 overexpression lines showed significant drought and cold stress tolerance at the seedling stage, we were prompted to determine whether the expression of ABA-regulated genes was also enhanced in these transgenic lines. Several ABAregulated genes ( $L E A 3, R A B 16 A$, and OsABA45) were selected, and its expressions levels between the control and OsPYL3 and OsPYL9 overexpression lines in response to ABA treatment were compared. Quantitative RT-PCR analysis indicated that ABA induced the expression of these genes, which was significantly higher than that observed in the controls (Fig. 8). Notably, in the absence of ABA treatment, the expression of these ABAregulated genes was still higher in the OsPYL3 and OsPYL9 overexpression lines than that in the control, indicating that the overexpression of OsPYL3 and OsPYL9 promoted the constitutive expression of ABA-regulated genes (Fig. 8), which possibly explains the delayed seed germination of OsPYL3 and OsPYL9 overexpression lines in the absence of ABA (Fig. 5c, d, and g). Taken together, the upregulation of ABA-regulated genes might also have contributed to the observed ABA hypersensitivity and the enhanced stress tolerance of the OsPYL3 and OsPYL9 overexpression lines.

\section{Discussion}

Members of the PYL protein family such as the ABA receptors and the core component in ABA-sensing signaling have been investigated in various plant species, including Arabidopsis, soybean, rice, and cucumber (Ma et al. 2009; Wang et al. 2012; Bai et al. 2013; He et al. 2014; Kim et al. 2014). Compared to the extensive studies on AtPYLs, the property, function, and mechanism of OsPYLs are largely unknown. In the present study, based on amino acid sequence analysis of AtPYLs and predicted OsPYLs in the rice genome annotation project, 10 out of the 13 OsPYLs were cloned and studied. OsPYLs were differentially expressed in the leaves, stems, roots, panicles, embryo, and endosperm, and some OsPYL members showed pronounced tissue-specific expression patterns (Fig. 1a). OsPYLs also displayed a differential response to ABA treatment (Fig. 1b). The diverse expression patterns of OsPYLs were indicative of their functional diversity. All OsPYLs were localized to the cytoplasm and nucleus (Fig. 2a), which was consistent with the results of previous reports on the subcellular localization of AtPYLs and GmPYLs (Ma et al. 2009; Bai et al. 2013).

The interaction between the OsPYLs and OsPP2Cs was investigated in both yeast and tobacco, and an overall interaction map was generated between 10 OsPYLs and 5 OsPP2C members in the absence or presence of ABA treatment (Figs. 3 and 4). OsPYLs selectively interacted with OsPP2Cs in ABA-dependent or ABA-independent manner. Combined with a recent report that OsPYLs inhibit OsPP2C activity ( $\mathrm{He}$ et al. 


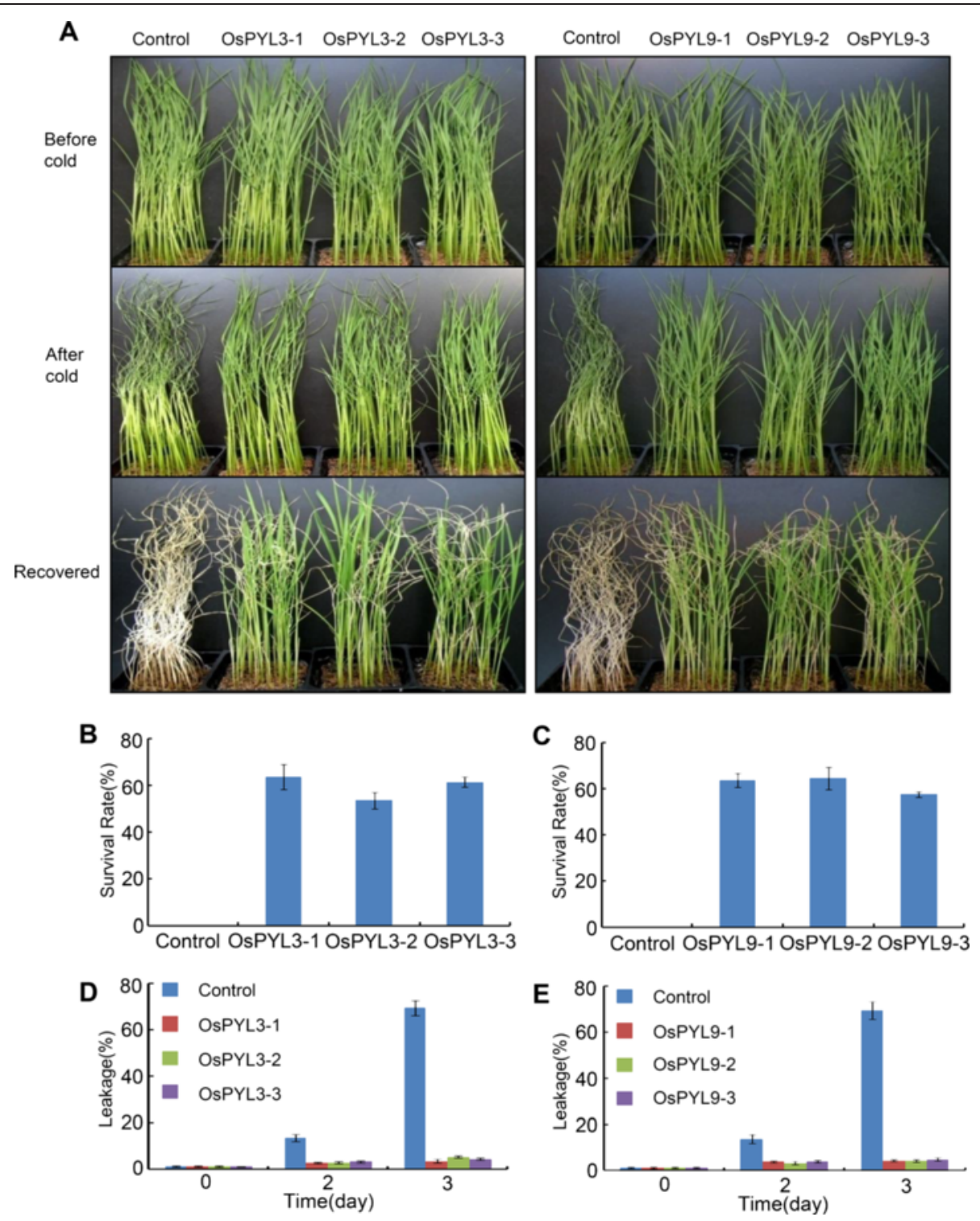

Fig. 7 Overexpression of OsPYL3 and OsPYL9 enhanced cold stress tolerance. a Two-week-old OSPYL3 and OsPYL9 overexpression seedlings were subjected to $10{ }^{\circ} \mathrm{C}$ treatment and then recovered at room temperature. Photographs were taken at before cold (upper panel) and 4 days after cold treatment (middle panel), and 7 days after recovered (bottom panel). $\mathbf{b}$ and $\mathbf{c}$ Survival rates of the cold-treated plants after 7 days of recovery. Values are mean \pm SD ( $n=30$ for each replicate) of three independent experiments. $\mathbf{d}$ and $\mathbf{e}$ Relative ion leakage in rice leaves after $10{ }^{\circ} \mathrm{C}$ treatment for $0 \mathrm{~d}, 2 \mathrm{~d}$ and $3 \mathrm{~d}$. Data represent means \pm SD of three biological replicates

2014), these data suggest that OsPYLs are functional rice ABA receptors.

In addition to the discovery of PYLs as ABA receptors, the present study has shown that these proteins also regulate ABA signaling and improve tolerance to abiotic stress. Overexpression of AtPYL4 and AtPYL5 leads to enhanced ABA hypersensitivity during seed germination and improved drought tolerance (Santiago et al. 2009; Pizzio et al. 2013). In addition, the rootspecific expression of AtPYL8 regulates ABA-mediated inhibition of root growth (Antoni et al. 2013). However, information on the biological function of OsPYLs in rice, which is considered a monocot model, is limited. OsPYL5 has been shown to positively regulate seed germination and drought stress response (Kim et al. 2012; Kim et al. 2014). In the present study, transgenic rice overexpressing OsPYL3 and OsPYL9 were produced and analyzed in detail (Fig. 5a and b). First, overexpression of OsPYL3 and OsPYL9 conferred ABA hypersensitivity during seed germination (Figs. 5c-i). In addition, overexpression of OsPYL3 and OsPYL9 in the absence of ABA significantly delayed seed germination, and OsPYL9 played more important roles, which was consistent with the upregulation of OsPYL9 in the embryo 

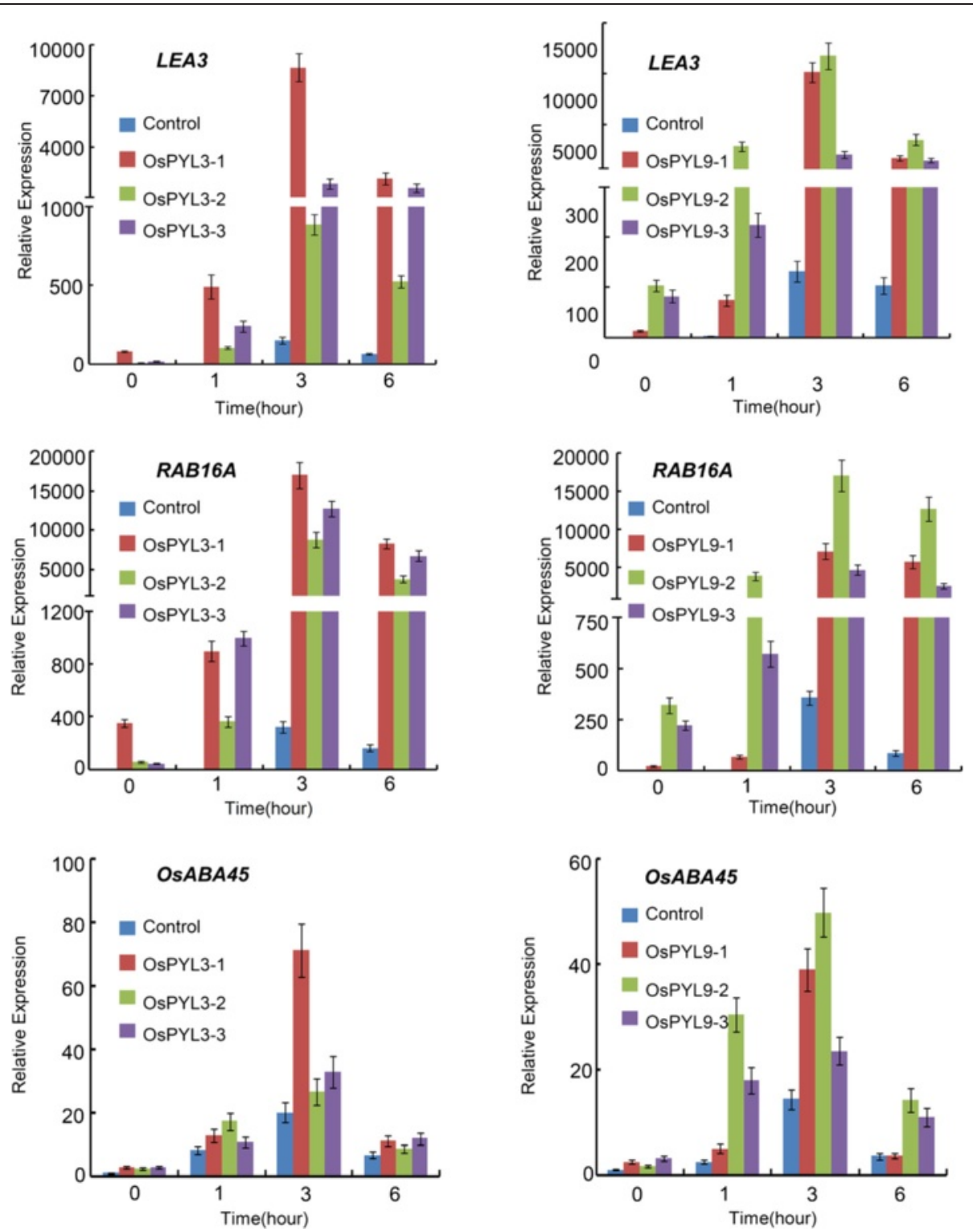

Fig. 8 Overexpression of OsPYL3 and OsPYL9 increased the expression of ABA responsive genes. Fourteen-day-old control, OsPYL3 and OsPYL9 overexpression seedlings were treated with ABA solution $(100 \mu \mathrm{M})$ for indicated time. Shoots were analyzed by quantitative RT-PCR. LEA3 (LOC_Os05946480), RAB16A (LOC_Os11926790), and OsABA45 (LOC_Os12g29400) were monitored. The expression level of ABA regulated gene in control at $0 \mathrm{~h}$ was set as 1 and the fold change was analyzed via the $2^{-\Delta \triangle C T}$ method using the rice ubiquitin gene as an internal control. Values represent the mean \pm SD of three biological replicates

compared to that of OsPYL9 (Fig. 5c-i). Second, overexpression of OsPYL3 and OsPYL9 enhanced drought stress tolerance, which can be partly explained by a slower water loss rate through the stomata in transgenic lines (Fig. 6). Third, OsPYL3 and OsPYL9 overexpression lines show increased membrane stability during cold stress and enhanced cold stress tolerance (Fig. 7). Last, expression of ABA-regulated genes in OsPYL3 and OsPYL9 overexpression lines was significantly higher than that in the controls (Fig. 8), which accounted for the increased stress tolerance of transgenic plants. Interestingly, compared to the controls, OsPYL3 and OsPYL9 overexpression lines did not show any negative effect on growth, grain yield, and other observable phenotypes, whereas these were observed in OsPYL5 (Kim et al. 2014). One possible explanation for this finding is that OsPYL5 was directed by the maize ubiquitin promoter, which is a very strong promoter in monocots, and OsPYL3 and OsPYL9 was directed by the $35 S$ promoter, whose efficiency was less than that of ubiquitin in rice. Taken together, these results indicate that OsPYL3 and OsPYL9 can be used as a target gene for the improvement of abiotic stress tolerance in rice. 


\section{Conclusions}

In the present study, we study the expression pattern of PYL orthologs in rice (OsPYL), generate an overall interaction map between 10 OsPYL members and 5 protein phosphatase $2 \mathrm{C}$ in rice (OsPP2C) members and show the biological function ofOsPYL3 and OsPYL9. Taken together, we comprehensively uncovered the properties of OsPYLs, which may be good candidates for the improvement of abiotic stress tolerance in rice.

\section{Methods}

\section{Plant materials, and rice transformation}

For cloning of OsPYLs and OsPP2Cs genes, Oryza sativa L. ssp. Japonica cv. Nipponbare was used. The coding sequence of OsPYLs and OsPP2Cs were cloned from the cDNA using standard PCR-based protocol. The primers used are listed at Additional file 1: Table S1. Full-length sequences of OsPYLs and OsPP2Cs were cloned into pENTR/D-Topo (Invitrogen), resultant constructs were confirmed by sequencing and saved for later use. To over express the OsPYLs in rice, OsPYLs was transferred to $p H 7 W G 2$ vector via $L R$ recombination reaction of the Gateway system. The resultant 35S:OsPYLs constructs, in which OsPYLs was drived by the cauliflower mosaic virus (CaMV) $35 S$ promoter, were transferred into Agrobacterium tumefaciens EHA105. Finally, OsPYLs were transformed into Oryza sativa L. ssp. Japonica cv. Longjing 11 by the Agrobacterium-mediated co-cultivation method.

\section{Yeast two-hybrid assay}

Full-length sequences of OsPYLs and OsPP2Cs were cloned into $p D E S T 32$ or $p D E S T 22$ vector via an LR recombination reaction and used as baits or preys respectively. The resultant constructs were transformed into the yeast strain $\mathrm{Y} 2 \mathrm{H}$ gold. Presence of the transgenes was confirmed by growth on a SD/-Leu/-Trp plate. To assess protein interactions, the transformed yeast cells were suspended in liquid SD/-Leu/-Trp to $\mathrm{OD}_{600}=1.0$. The suspended cells were spread on plates containing $\mathrm{SD} /$-His/-Leu/-Trp medium supplied with indicated concentration of 3-AT (3-amino-1, 2, 4-triazole) and ABA. The interactions were observed after 4 days of incubation at $30{ }^{\circ} \mathrm{C}$. The experiments were repeated three times with similar results.

\section{Subcellular localization and Bimolecular fluorescence complementation in tobacco}

For subcellular localization of OsPYLs and OsPP2Cs, fulllength sequences of OsPYLs and OsPP2Cs were cloned into the $\mathrm{pH} 7 \mathrm{WGF}$ vector via an LR recombination reaction, in which the OsPYLs and OsPP2Cs fused with green fluorescent protein (GFP) was drived by $35 \mathrm{~S}$ promoter. For Bimolecular fluorescence complementation (BiFC) assays, OsPYLs were fused with the C-terminal portion of the yellow fluorescent protein (YFP) via an LR recombination reaction; OsPP2Cs were fused with the $\mathrm{N}$-terminal portion of the yellow fluorescent protein (YFP) via an LR recombination reaction. For transient expression, the fusion constructs were transferred into Agrobacterium tumefaciens GV3101. Agrobacterium tumefaciens strain harboring each construct along with the $p 19$ strain were infiltrated into 4-week-old $N$. benthamiana leaves. For staining of the nuclei, $10 \mathrm{mg} / \mathrm{ml} \mathrm{4,} \mathrm{6-diamidino-2-pheny-}$ lindole (DAPI) was infiltrated into $N$. benthamiana leaves $3 \mathrm{~h}$ before observation. For microscopic analyses, leaf discs were cut $3 \mathrm{~d}$ after infiltration. The fluorescence signal was observed using confocal microscopy.

\section{Analysis of genes expression}

Total RNA was extracted using TRIzol (Invitrogen) and treated with DNaseI. cDNA was synthesized from $2 \mu \mathrm{g}$ of total RNA using SuperscriptII Reverse Transcriptase. Real-time PCR was performed with SYBR Green PCR master mix (TransStart). Data were collected using BioRad chromo 4 real-time PCR detector. All expressions were normalized against the Ubiquitin gene. The primers used are listed at Additional file 1: Table S1.

\section{Germination and abiotic stress tolerance assay}

Dehulled seeds were surface-sterilized and planted on half-strength MS medium supplemented with indicated concentration of ABA (A1049, Sigma-Aldrich). Seed germination was defined as the coleoptiles emerged from the seed and scored every $12 \mathrm{~h}$ for 5 days. Three independent T3 homologous transgenic lines and the control Longjing 11 were used for stress tolerance experiments. For the dehydration treatment, rice plants grown for 3 weeks were withhold water for 10 days and then rehydrated and grown under normal conditions for 7 days. For cold treatment, 14-day-old seedlings were transferred to $10{ }^{\circ} \mathrm{C}$ for 4 days and then returned to normal growth conditions for 7 days. The survival rates were recorded. Approximately 50 seedlings of each line were used for each experiment, and three replicates of each experiment were performed. Tests for statistical analysis between transgenic lines and the controls were performed using Microsoft excel 2007.

\section{Water loss assay and measurement of relative electrolyte leakage}

For water loss assay, leaves of control and OsPYLs transgenic plants grown under normal conditions were detached from 3-week-old seedlings and weighed immediately on a piece of weighing paper, and then placed on a laboratory table and weighed at indicated time intervals. Three replicates were performed for each line. 
For relative water content measurement (RWC), Three-week-old seedlings of control and three independent OsPYLs transgenic lines were withhold water. The protocol was as described by (Barrs and Weatherley 1962). RWC was measured until the leaf cannot expand during dipped in the water.

Two-week-old seedlings of control and three independent OsPYLs transgenic lines were transferred into a $10{ }^{\circ} \mathrm{C}$ chamber. At $0 \mathrm{~d}, 2 \mathrm{~d}$ and $3 \mathrm{~d}, 0.5 \mathrm{~g}$ of leaves were harvested from each of ten plants. Leaf fragments were immersed in $6 \mathrm{~mL}$ deionized water and shaken at 100 rpm at $25{ }^{\circ} \mathrm{C}$ for $2 \mathrm{~h}$, and electrical conductivity was determined (C1). The samples were then boiled for 20 min, and the total conductivity was determined again (C2) after cooling to room temperature. Relative ion leakage (\%) was calculated as $\mathrm{C} 1 / \mathrm{C} 2 \times 100$.

\section{Additional file}

Additional file 1: Figure S1. Interaction of OsPYLs and OsPP2Cs in yeast two-hybrid. Figure S2. Pylogenetic analysis of PYLs family members in Rice and Arabidopsis. Table S1. Primers used in this study. (DOCX $8631 \mathrm{~kb}$ )

\section{Competing interests}

The authors declare that they have no competing interests.

\section{Authors' contributions}

$\mathrm{XT}$ and ZW performed most of experiments on vectors construction and biochemical assays. XL, TL, HL, and LW worked on the drought and cold response analysis. HN worked on rice transformation. QB conceived the project and corrected the manuscript. All of the authors read and approved the final manuscript.

\section{Acknowledgements}

This work was supported by Hundred-Talent-Program of Chinese Academy of Sciences, National Natural Science Foundation of China (No. 31070255), Excellent Academic Leaders of Harbin (RC2014XK002003), National Key Project of Transgenic Crops of China (2014ZX0800205B-003) and the 863 High Tech Program of Ministry of Science and Technology of China (2012AA101105)

\section{Author details}

Northeast Institute of Geography and Agroecology, Key Laboratory of Soybean Molecular Design Breeding, Chinese Academy of Sciences, Harbin 150081, China. ${ }^{2}$ Graduate University of Chinese Academy of Sciences, Beijing 100049, China. ${ }^{3}$ Rice Research Institute, Heilongjiang Academy of Land Reclamation Sciences, Jiamusi 154007, China. ${ }^{4}$ Cultivation institute, Heilongjiang Academy of Agricultural Science, Harbin 150086, China. ${ }^{5}$ National Engineering Research Centre for Wheat, Henan Agricultural University, Zhengzhou 450002, China.

Received: 13 February 2015 Accepted: 23 July 2015

Published online: 11 September 2015

\section{References}

Antoni R, Gonzalez-Guzman M, Rodriguez L, Peirats-Llobet M, Pizzio GA, Fernandez MA, De Winne N, De Jaeger G, Dietrich D, Bennett MJ et al (2013) PYRABACTIN RESISTANCE1-LIKE8 plays an important role for the regulation of abscisic acid signaling in root. Plant Physiol 161(2):931-941

Bai G, Yang DH, Zhao Y, Ha S, Yang F, Ma J, Gao XS, Wang ZM, Zhu JK (2013) Interactions between soybean $\mathrm{ABA}$ receptors and type $2 \mathrm{C}$ protein phosphatases. Plant Mol Biol 83(6):651-664
Barrs HD, Weatherley PE (1962) A Re-examination of the relative turgidity technique for estimating water deficits in leaves. Aust J Biol Sci 15:413-428

Cutler SR, Rodriguez PL, Finkelstein RR, Abrams SR (2010) Abscisic acid: emergence of a core signaling network. Annu Rev Plant Biol 61:651-679

Gonzalez-Guzman M, Pizzio GA, Antoni R, Vera-Sirera F, Merilo E, Bassel GW, Fernandez MA, Holdsworth MJ, Perez-Amador MA, Kollist H et al (2012) Arabidopsis PYR/PYL/RCAR receptors play a major role in quantitative regulation of stomatal aperture and transcriptional response to abscisic acid. Plant Cell 24(6):2483-2496

Gonzalez-Guzman M, Rodriguez L, Lorenzo-Orts L, Pons C, Sarrion-Perdigones A, Fernandez MA, Peirats-Llobet M, Forment J, Moreno-Alvero M, Cutler SR et al (2014) Tomato PYR/PYL/RCAR abscisic acid receptors show high expression in root, differential sensitivity to the abscisic acid agonist quinabactin, and the capability to enhance plant drought resistance. J Exp Bot 65(15): 4451-4464

Hao Q, Yin P, Li W, Wang L, Yan C, Lin Z, Wu JZ, Wang J, Yan SF, Yan N (2011) The molecular basis of ABA-independent inhibition of PP2Cs by a subclass of PYL proteins. Mol Cell 42(5):662-672

He Y, Hao Q, Li W, Yan C, Yan N, Yin P (2014) Identification and characterization of ABA receptors in Oryza sativa. PLoS One 9(4):e95246

Joshi-Saha A, Valon C, Leung J (2011) Abscisic acid signal off the STARting block. Mol Plant 4(4):562-580

Kim H, Hwang H, Hong JW, Lee YN, Ahn IP, Yoon IS, Yoo SD, Lee S, Lee SC, Kim $B G$ (2012) A rice orthologue of the ABA receptor, OsPYL/RCAR5, is a positive regulator of the $A B A$ signal transduction pathway in seed germination and early seedling growth. J Exp Bot 63(2):1013-1024

Kim H, Lee K, Hwang H, Bhatnagar N, Kim DY, Yoon IS, Byun MO, Kim ST, Jung KH, Kim BG (2014) Overexpression of PYL5 in rice enhances drought tolerance, inhibits growth, and modulates gene expression. J Exp Bot 65(2):453-464

Klingler JP, Batelli G, Zhu JK (2010) ABA receptors: the START of a new paradigm in phytohormone signalling. J Exp Bot 61(12):3199-3210

Li W, Wang L, Sheng X, Yan C, Zhou R, Hang J, Yin P, Yan N (2013) Molecular basis for the selective and ABA-independent inhibition of PP2CA by PYL13. Cell Res 23(12):1369-1379

Ma Y, Szostkiewicz I, Korte A, Moes D, Yang Y, Christmann A, Grill E (2009) Regulators of PP2C phosphatase activity function as abscisic acid sensors. Science 324(5930):1064-1068

Melcher K, Ng LM, Zhou XE, Soon FF, Xu Y, Suino-Powell KM, Park SY, Weiner JJ, Fujii H, Chinnusamy V et al (2009) A gate-latch-lock mechanism for hormone signalling by abscisic acid receptors. Nature 462(7273):602-608

Park SY, Fung P, Nishimura N, Jensen DR, Fujii H, Zhao Y, Lumba S, Santiago J, Rodrigues A, Chow TF et al (2009) Abscisic acid inhibits type 2C protein phosphatases via the PYR/PYL family of START proteins. Science 324(5930):1068-1071

Pizzio GA, Rodriguez L, Antoni R, Gonzalez-Guzman M, Yunta C, Merilo E, Kollist H, Albert A, Rodriguez PL (2013) The PYL4 A194T mutant uncovers a key role of PYR1-LIKE4/PROTEIN PHOSPHATASE 2CA interaction for abscisic acid signaling and plant drought resistance. Plant Physiol 163(1):441-455

Santiago J, Rodriques A, Saez A, Rubio S, Antoni R, Dupeux F, Park SY, Marquez JA, Cutler SR, Rodriguez PL (2009) Modulation of drought resistance by the abscisic acid receptor PYL5 through inhibition of clade A PP2Cs. Plant J 60(4):575-588

Wang Y, Wu Y, Duan C, Chen P, Li Q, Dai S, Sun L, Ji K, Sun Y, Xu W et al (2012) The expression profiling of the CsPYL, CsPP2C and CsSnRK2 gene families during fruit development and drought stress in cucumber. J Plant Physiol 169(18):1874-1882

Xue T, Wang D, Zhang S, Ehlting J, Ni F, Jakab S, Zheng C, Zhong Y (2008) Genome-wide and expression analysis of protein phosphatase $2 \mathrm{C}$ in rice and Arabidopsis. BMC Genomics 9:550

Zhao Y, Chan Z, Xing L, Liu X, Hou YJ, Chinnusamy V, Wang P, Duan C, Zhu JK (2013) The unique mode of action of a divergent member of the ABA-receptor protein family in ABA and stress signaling. Cell Res 23(12):1380-1395 